\section{On the Extended-Born Technique for Scattering from Buried Dielectric Targets}

Nilanjan Dasgupta, Norbert Geng,

Traian Dogaru, and Lawrence Carin

\begin{abstract}
The extended Born technique is an approximate nonlinear method for analyzing scattering from a weak discontinuity. Moreover, when applied to the low-frequency (electromagnetic induction) applications for which it was developed originally, extended Born has accurately modeled scattering from inhomogeneities considerably larger than those appropriate for the standard linear Born technique. In this letter, we examine the extended Born technique at radar frequencies, considering three-dimensional (3-D) scattering from a dielectric target buried in a lossy half space.
\end{abstract}

Index Terms-Born approximation, buried object detection.

There are many applications for which one would like to consider electromagnetic scattering from a weak inhomogeneity in a background medium. For example, plastic anti-personnel land mines are often composed of materials with dielectric constants close to those of the background soil [1]. Such a scattering problem can be solved rigorously via the method of moments (MoM) [2], taking proper account of the half-space or layered-medium Green's function [2], [3] used to model the soil. However, while the MoM is highly accurate, it is often computationally expensive. In the context of the weak-scattering problem of interest here, we can avail ourselves of approximate modeling algorithms, which can yield accurate results while simultaneously being relatively inexpensive computationally. The Born approximation [4] is a well-known example of such a technique, it assuming that the fields inside the scatterer are the same as the incident fields, in the absence of the inhomogeneity. While this is a popular technique, it is only appropriate for relatively small inhomogeneities [4], [5]. Recently, Habashy et al. [5] have developed a new algorithm, which they demonstrated can handle inhomogeneities considerably larger than appropriate for the Born approximation. However, their previous research concentrated on very-low-frequency (kilohertz) applications, of interest in geophysics [5]. Moreover, previous results assumed a homogeneous background medium. Here we consider radar-frequency operation, for a target buried in a half-space.

Consider a nonmagnetic background medium characterized by the generally inhomogeneous permittivity $\epsilon_{b}(\boldsymbol{r})$. Further, assume that the permittivity $\epsilon(\boldsymbol{r})$ characterizes the medium when a dielectric inhomogeneity is present (i.e., $\epsilon(\boldsymbol{r})-\epsilon_{b}(\boldsymbol{r}) \neq 0$ for $\boldsymbol{r} \in V$, where $V$ is the volume of the discontinuity, while $\epsilon(\boldsymbol{r})-\epsilon_{b}(\boldsymbol{r})=0$ for $\boldsymbol{r} \notin V)$. If $\boldsymbol{G}\left(\boldsymbol{r}, \boldsymbol{r}^{\prime}\right)$ characterizes the dyadic Green's function for the medium $\epsilon_{b}(\boldsymbol{r})$, then the total electric field at $\boldsymbol{r}$ is [4], [5]

$$
\boldsymbol{E}(\boldsymbol{r})=\boldsymbol{E}^{i n c}(\boldsymbol{r})+\int_{V} \boldsymbol{G}\left(\boldsymbol{r}, \boldsymbol{r}^{\prime}\right) \cdot \boldsymbol{E}\left(\boldsymbol{r}^{\prime}\right)\left[\epsilon\left(\boldsymbol{r}^{\prime}\right)-\epsilon_{b}\left(\boldsymbol{r}^{\prime}\right)\right] d^{3} \boldsymbol{r}^{\prime} .
$$

The well-known Born approximation is characterized by assuming $\boldsymbol{E}\left(\boldsymbol{r}^{\prime}\right) \approx \boldsymbol{E}^{\text {inc }}\left(\boldsymbol{r}^{\prime}\right)$ inside the integral, which yields a direct but

Manuscript received October 27, 1998; revised August 23, 1999.

N. Dasgupta, T. Dogaru, and L. Carin are with the Department of Electrical and Computer Engineering, Duke University, Durham, NC 27708-0291 USA.

N. Geng was with the Department of Electrical and Computer Engineering, Duke University, Durham, NC 27708-0291 USA. He is now with the University of Karlsruhe, Institut fuer Hoechstfrequenztechnik und Elektronik D-76128 Karlsruhe, Germany.

Publisher Item Identifier S 0018-926X(99)09951-2.

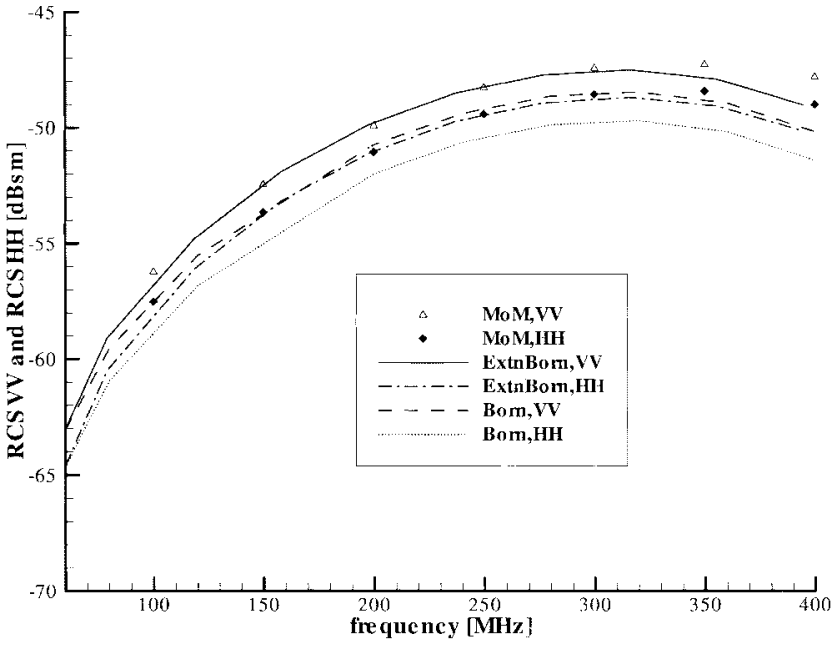

Fig. 1. Backscattered radar cross section (RCS) of a $10-\mathrm{cm}$ radius sphere with its center placed $15 \mathrm{~cm}$ beneath the air-ground interface due to vertically $(\mathrm{V})$ or horizontally $(\mathrm{H})$ polarized plane-wave excitation, $50^{\circ}$ from grazing. The lossless sphere is characterized by dielectric constant $\epsilon_{r}=3$ and the lossy background soil is characterized by $\epsilon_{r}=4$ and $\sigma=0.01 \mathrm{~S} / \mathrm{m}$.

approximate solution for $\boldsymbol{E}(\boldsymbol{r})$. This solution is linear in the inhomogeneity $\epsilon(\boldsymbol{r})-\epsilon_{b}(\boldsymbol{r})$, which implies that it neglects multibounce interactions inside the scatterer [4], [5].

The extended Born approximation is based on recognizing that $\boldsymbol{G}\left(\boldsymbol{r}, \boldsymbol{r}^{\prime}\right)$ is large for $\boldsymbol{r}$ in the vicinity of $\boldsymbol{r}^{\prime}$, while being relatively small for $\boldsymbol{r}$ distant from $\boldsymbol{r}^{\prime}$. Consequently,

$$
\begin{aligned}
\boldsymbol{E}(\boldsymbol{r}) \approx & \boldsymbol{E}^{i n c}(\boldsymbol{r})+\int_{V} \boldsymbol{G}\left(\boldsymbol{r}, \boldsymbol{r}^{\prime}\right) \\
& \cdot \boldsymbol{E}(\boldsymbol{r})\left[\epsilon\left(\boldsymbol{r}^{\prime}\right)-\epsilon_{b}\left(\boldsymbol{r}^{\prime}\right)\right] d^{3} \boldsymbol{r}^{\prime}, \quad \text { for } \boldsymbol{r} \in V .
\end{aligned}
$$

Rearranging (2), we find

$$
\begin{aligned}
\boldsymbol{E}(\boldsymbol{r}) & \approx \boldsymbol{M}(\boldsymbol{r})^{-1} \cdot \boldsymbol{E}^{i n c}(\boldsymbol{r}), \quad \text { for } \boldsymbol{r} \in V \\
\boldsymbol{M}(\boldsymbol{r}) & \equiv \boldsymbol{I}-\int_{V} \boldsymbol{G}\left(\boldsymbol{r}, \boldsymbol{r}^{\prime}\right)\left[\epsilon\left(\boldsymbol{r}^{\prime}\right)-\epsilon_{b}\left(\boldsymbol{r}^{\prime}\right)\right] d^{3} \boldsymbol{r}^{\prime} .
\end{aligned}
$$

The expression in (3) yields an approximation for the electric fields inside the target, which can be used in (1) to find the fields everywhere (i.e., for $\boldsymbol{r} \notin V$ ); this approximation has been termed "extended Born" [5]. Note that the inverse of $M$ approximately accounts for multiple interactions, yielding a nonlinear algorithm in $\epsilon(\boldsymbol{r})-\epsilon_{b}(\boldsymbol{r})$.

If the integral term in (3) is ignored, extended Born reduces to the classical Born approximation. Moreover, the extended Born approximation involves the inversion of a $3 \times 3$ matrix $\boldsymbol{M}(\boldsymbol{r})$, for each $r \in V$. These matrices are computed by representing $\epsilon(\boldsymbol{r})-\epsilon_{b}(\boldsymbol{r})$ as an aggregate of $N$ three-dimensional cubes (or other appropriate basis function) [5], with cube dimensions small relative to wavelength; here the cubes have dimensions of at most $\lambda / 10$, where $\lambda$ is the wavelength in the medium for which $\epsilon(\boldsymbol{r})-\epsilon_{b}(\boldsymbol{r}) \neq 0$. We compute $\boldsymbol{M}(\boldsymbol{r})$ at the $N \boldsymbol{r}_{n}$ that constitute the centers of the cubes. Consequently, we need invert $N 3 \times 3$ matrices. This can be contrasted with a volumetric MoM solution of (1), in which the fields $\boldsymbol{E}\left(\boldsymbol{r}^{\prime}\right), \boldsymbol{r}^{\prime} \in V$ are expanded in a basis. The basis functions must again be small relative to wavelength, but in the case of MoM the constituent to be expanded is a vector, in comparison to the scalar $\epsilon(\boldsymbol{r})-\epsilon_{b}(\boldsymbol{r})$. Moreover, in a volumetric MoM solution of this same 


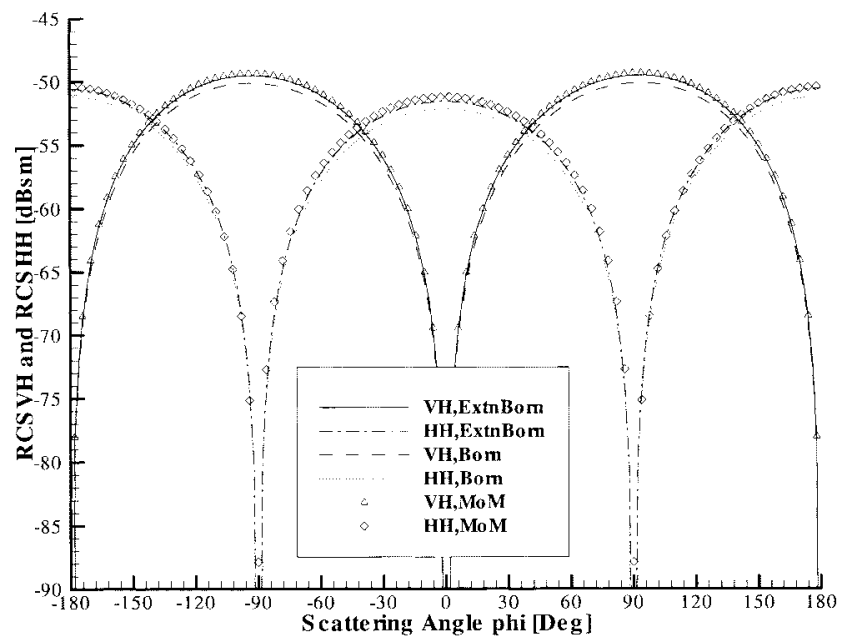

(a)

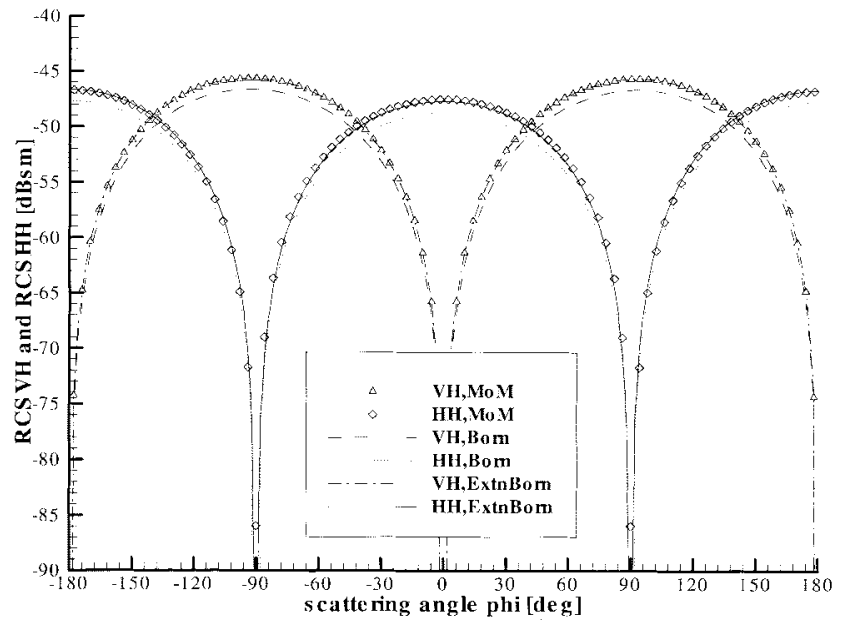

(b)

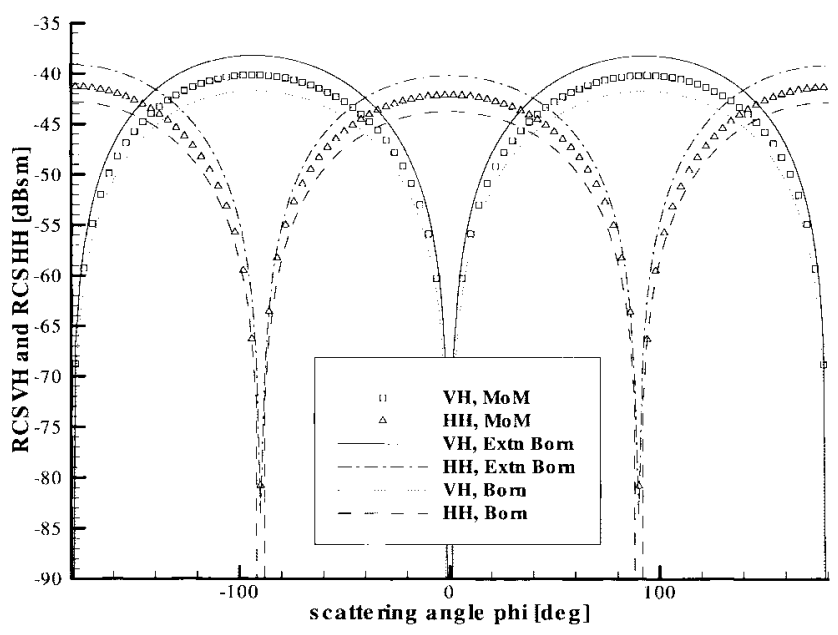

(c)

Fig. 2. Bistatic RCS of a $10-\mathrm{cm}$ radius sphere with its center placed $15 \mathrm{~cm}$ beneath the air-ground interface. The incident plane wave is incident $50^{\circ}$ from grazing at $\phi=0^{\circ}$ and bistatic-scattering results are plotted as a function of $\phi, 50^{\circ}$ from grazing. The lossy background soil is characterized by $\epsilon_{r}=4$ and $\sigma=0.01 \mathrm{~S} / \mathrm{m}$ and the operating frequency is $300 \mathrm{MHz}$ corresponding to $d / \lambda=0.4$, where $d$ is the sphere diameter and $\lambda$ is the wavelength in the soil. Results are plotted for variable dielectric constants for the lossless sphere. Sphere dielectric constant (a) $\epsilon_{r}=3.5,(\mathrm{~b}) \epsilon_{r}=3.0$, and (c) $\epsilon_{r}=2.0$.

problem, we invert a single $3 N \times 3 N$ (three vector field components, each represented by a separate cube), rather than $N 3 \times 3$ matrices.

As with the MoM, the extended Born technique requires accurate computation of the dyadic Green's function $\boldsymbol{G}\left(\boldsymbol{r}, \boldsymbol{r}^{\prime}\right)$. While this is relatively straightforward for the free-space case [4], [5], for the layered-medium problem of interest here, accurate and efficient computation of $\boldsymbol{G}\left(\boldsymbol{r}, \boldsymbol{r}^{\prime}\right)$ is challenging. We have utilized the compleximage technique, which represents the dyadic Green's function as a sum of homogeneous-medium Green's functions with generally complex source points [6].

Below we present a series of results for a lossless dielectric target buried in a lossy half-space. In addition to the results computed via Born and extended Born, reference MoM results are presented. The MoM results were computed via a surface-integral-equation formulation (rather than the volumetric MoM procedure alluded to above). A detailed discussion of this MoM formulation can be found in [2] and [7].

Before proceeding, we note that the loss in the half-space is used to represent the characteristics of soil, but it is also critical to the accuracy of the extended Born algorithm. In particular, recall that the approximation in (2) (which drives the extended-Born algorithm) is based on the assumption that $\boldsymbol{G}\left(\boldsymbol{r}, \boldsymbol{r}^{\prime}\right)$ is peaked near $\boldsymbol{r}=\boldsymbol{r}^{\prime}$ and decays quickly with increasing $\left|\boldsymbol{r}-\boldsymbol{r}^{\prime}\right|$. Essentially, this implies that the fields due to a point source at $\boldsymbol{r}^{\prime}$ diminish quickly with increasing $\left|\boldsymbol{r}-\boldsymbol{r}^{\prime}\right|$; this phenomenon is aided by the presence of loss in the background medium in which the discontinuity is immersed. In particular, we tested the extended Born algorithm for a lossless background medium (e.g., vacuum), at the radar frequencies of interest-for this case, we did not see any advantage of the extended Born algorithm vis-à-vis conventional linear Born.

The first set of results consider a sphere of $10-\mathrm{cm}$ radius and dielectric constant $\epsilon_{r}=3.0$, buried in a half-space with dielectric constant $\epsilon_{r}=4.0$ and conductivity $\sigma=0.01 \mathrm{~S} / \mathrm{m}$. The center of the sphere is buried $15 \mathrm{~cm}$ from the air-ground interface, and the incidence angle is $50^{\circ}$ from grazing. Far-zone backscatter results are shown in Fig. 1, for $\mathrm{VV}$ and $\mathrm{HH}$ polarization (vertical polarization incidence, vertical-polarization receive; horizontal-polarization incidence, and horizontal-polarization receive). For this symmetric target, there are no cross-polarized backscattered fields [8]. Results are plotted as a function of operating frequency and we see that the Born and extended Born solutions are in close agreement at frequencies less than approximately $100 \mathrm{MHz}(d / \lambda<0.137$, where 
$\lambda$ is the approximate wavelength in the lossy soil and $d$ is the sphere diameter), while the extended Born solution stays in close agreement with the MoM results up to approximately $300 \mathrm{MHz}$ (i.e., for $d / \lambda \leq 0.4$ ). These results demonstrate the superiority of extended Born relative to conventional Born. In particular, for a fixed inhomogeneity, these results indicate that extended Born allows consideration of larger targets (electrically), implying accurate performance at higher operating frequencies.

The results in Fig. 1 considered a single inhomogeneity profile at variable frequencies. To further address the accuracy of the extended Born and Born algorithms relative to MoM, we fix the operating frequency at $300 \mathrm{MHz}$ and vary the target-induced inhomogeneity for the same half-space background. The sphere radius is again $10 \mathrm{~cm}$ placed $15 \mathrm{~cm}$ from the interface; the target is situated close to the surface to enhance target-surface interaction (this being handled rigorously via MoM) approximately via extended Born, and not at all via classical Born. In Fig. 2(a)-(c), we consider the target with dielectric constant 3.5, 3.0, and 2.0 (recall the soil has a dielectric constant $\epsilon_{r}=4$ and a conductivity of $\sigma=0.01 \mathrm{~S} / \mathrm{m}$ ). The farzone bistatic results are plotted as a function of the azimuthal angle $\phi, 50^{\circ}$ from grazing, for an incident field propagating $50^{\circ}$ from grazing, at $\phi=0^{\circ}$. For a target of dielectric constant $\epsilon_{r}=3.5$ and $\epsilon_{r}=3.0$, the agreement between extended Born and the MoM is excellent for all bistatic angles. By comparison, for these same cases a noticeable discrepancy $(1 \mathrm{~dB})$ is seen between MoM and classical Born, attributed to the degree of inhomogeneity as well as the proximity of the target to the interface. For this example, we see that the extended Born breaks down appreciably when the target dielectric constant is reduced further to $\epsilon_{r}=2$ [Fig. 2(c)]. Finally, close inspection of Fig. 2(a) and (b) implies that the extended Born is more accurate for a target with $\epsilon_{r}=3.0$, relative to $\epsilon_{r}=3.5$. We attribute this anomaly to inaccuracies in the MoM rather than in extended Born. In particular, it has been mentioned that the surfaceintegral-equation MoM formulation used here can often be slightly inaccurate for very weak inhomogeneities [9]. Finally, while space limitations prohibit our showing the results here, the extended Bornpredicted VV fields were found to be as accurate as the $\mathrm{HH}$ and $\mathrm{VH}$ results in Fig. 2.

It is of interest to address the memory and central processing unit (CPU) requirements of the extended Born solution, relative to the Born and surface MoM solutions shown here for comparison. In Fig. 3, we plot the CPU and RAM requirements of the extended Born, Born, and MoM solutions for the problem in Fig. 1. With regard to the MoM solution, results are shown for a general triangular-patch model for the surface currents [7] and, in all results, the subsectional basis functions were designed to discretizate at a level of 20 basis functions per wavelength (for all methods). As expected, the simple Born solution requires the least CPU and memory. However, it is also important to note that the extended Born solution, which provides very accurate results relative to the benchmark MoM solution (see Fig. 1), requires significantly less CPU and RAM than the MoM. The electrical size of the sphere surface area scales as $f^{2}\left(\operatorname{order}(r / \lambda)^{2}\right.$, where $r$ is the constant sphere radius and $\lambda$ the variable wavelength), as does the number of patch basis functions $N$ required in the surface MoM solution, where $f$ is the frequency. Consequently, the MoM RAM is of order $f^{4}$ (if $N$ is the number of unknowns, the RAM required for the MoM impedance matrix is order $N^{2}$ ). Further, at low frequencies the MoM CPU is order $f^{4}$ required to fill the $N \times N$ MoM impedance matrix, with this becoming order $f^{6}$ at higher frequencies, where the LU decomposition matrix solver (order $N^{3}$ ) becomes the primary computational burden. The memory requirements of the extended Born solution are almost frequency independent, since for all frequencies we need only store consecutive $3 \times 3$ matrices. At

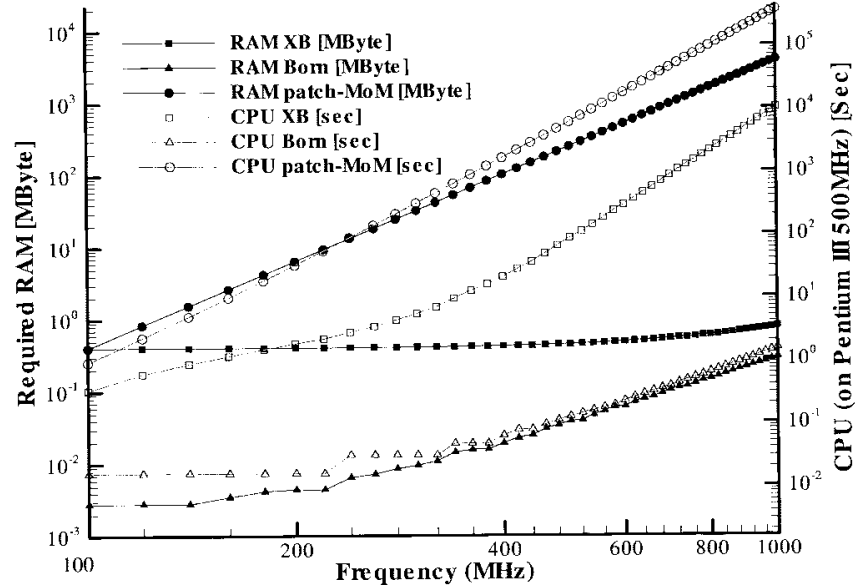

Fig. 3. Comparison of the RAM and CPU requirements of the extended Born, Born, and general triangular-patch method-of-moments (patch MOM) solutions [7], for the example in Fig. 1. All results were run on a 500-MHz Pentium III personal computer.

low frequencies the extended Born CPU is approximately linear in the number of unknowns and, therefore, is of order $f^{3}$ (extended Born is a volumetric method vis-à-vis the surface MoM solution). Note that as the frequency increases, the slope of the extended Born CPU changes. We attribute this to the computational burden of computing the half-space Green's function at higher frequencies [2], [7].

In conclusion, the extended Born algorithm has been utilized to model electromagnetic scattering at radar frequencies for dielectric targets buried in a lossy half-space. In general, for the class of problems considered here, the extended Born algorithm yields results that are at least as accurate as conventional Born, and often the results are considerably better (relative to a rigorous MoM solution). However, the computational complexity of extended Born is higher than that of classical Born, since the extended-Born algorithm requires accurate computation of the background medium (half-space) Green's function, as well as multiple volumetric near-field integrations. We also note that we have focused on frequency-domain integral-equation formulations, although for time-domain applications, the finite difference time domain may be more appropriate [10], it also applicable to a more general problem class (not just layered media). Finally, all results presented here have been for far-zone scattering. Future research will involve consideration of extended Born for near-zone radar scattering in a half space. Such an extended-Born solution has been successfully implemented for electromagnetic induction (EMI) problems [11].

\section{REFERENCES}

[1] L. Carin, N. Geng, M. McClure, J. Sichina, and L. Nguyen, "Ultrawideband synthetic aperture radar for mine-field detection," IEEE Antennas Propagat. Mag., vol. 41, pp. 18-33, Feb. 1999.

[2] N. Geng and L. Carin, "Wideband electromagnetic scattering from a dielectric body of revolution buried in a lossy, dispersive layered medium," IEEE Trans. Antennas Propagat., vol. 47, pp. 610-619, Apr. 1999.

[3] K. A. Michalski and D. Zheng, "Electromagnetic scattering and radiation by surfaces of arbitrary shape in layered media, parts I and II," IEEE Trans. Antennas Propagat., vol. 38, pp. 335-352, Mar. 1990.

[4] W. C. Chew, Waves and Fields in Inhomogeneous Media. Oxford, U.K.: Oxford Press, 1996.

[5] T. M. Habashy, R. W. Groom, and B. Spies, "Beyond the Born and Rytov approximations: A nonlinear approach to electromagnetic scattering," J. Geophys. Res., vol. 98, pp. 1759-1775, 1993.

[6] R. M. Shubair and Y. L. Chow, "A simple and accurate complex image interpretation of vertical antennas present in contiguous dielectric half- 
spaces," IEEE Trans. Antennas Propagat., vol. 41, pp. 806-812, June 1993.

[7] J. He, N. Geng, T. Yu, and L. Carin, "Electromagnetic scattering from a general dielectric target embedded in a multilayered medium," Radio Sci. to be published.

[8] L. Carin, R. Kapoor, and C. E. Baum, "A Polarimetric SAR imaging of buried landmines," IEEE Trans. Geosci. Remote Sensing, vol. 36, pp. 1985-1988, Nov. 1998.

[9] J. R. Mautz and R. F. Harrington, "Electromagnetic scattering from a homogeneous material body of revolution," $A E U$, vol. 33, pp. 71-80, 1979.

[10] J. M. Bourgeois and G. S. Smith, "A fully three-dimensional simulation of a ground-penetrating radar: FDTD theory compared with measurements," IEEE Trans. Geosci. Remote Sensing, vol. 34, pp. 36-44, Jan. 1996.

[11] T. Yu and L. Carin, "Extended-Born analysis of the electromagneticinduction response of a dielectric target embedded in a lossy layered medium," Radio Sci., to be published.

\section{Buried Object Detection and Location Estimation from Electromagnetic Field Measurements}

\author{
George A. Tsihrintzis, Peter Meincke Johansen, \\ and Anthony J. Devaney
}

\begin{abstract}
A translation property is derived describing the field scattered from a known buried object placed at distinct locations. The result is used to derive the optimum algorithm for detecting the known buried object and estimating its location from noisy scattered electromagnetic field measurements.
\end{abstract}

Index Terms-Ground penetrating radar, Lippmann-Schwinger equation, object detection, wave scattering.

\section{INTRODUCTION}

The problem of detecting known buried objects and estimating their location from electromagnetic field measurements is relevant in many technological areas such as demining, buried waste clean up, excavation planning, and archaeological investigations. In all of the above applications, serious challenges arise, mainly due to physical limitations such as: 1) significant losses due to moist soil that limit the signal-to-noise ratio; 2) presence of a large number of randomly distributed unwanted objects, returns from which obscure the return from the object of interest (volume clutter); and 3) random roughness of the air/soil interface that results in incoherent (random) returns (surface clutter). As a result of these limitations, standard threshold detection algorithms [1] may not successfully address the buried object detection problem, especially when the object is small and the return signal weak.

Manuscript received August 27, 1998; revised August 16, 1999. The work of G. A. Tsihrintzis and A. J. Devaney were supported in part by ARO Grant DAAG55-97-1-0013 and by AFOSR Grant F49620-96-1-0028. The work of P. M. Johansen was supported by the Danish Technical Research Council.

G. A. Tsihrintzis is with the Department of Informatics, University of Piraeus, Piraeus, 18534 Greece.

P. M. Johansen is with the Department of Electromagnetic Systems, Technical University of Denmark, Lyngby, DK-2800 Denmark.

A. J. Devaney is with the Department of Electrical and Computer Engineering, Northeastern University, Boston, MA 02115, USA.

Publisher Item Identifier S 0018-926X(99)09952-4.
The signal processing strategy with the highest potential to address the buried object detection problem is the one that utilizes field models for the air/soil/object environment. Due to the complicated nature of this environment, however, certain simplifications need to be made, namely the assumption of a planar air/soil interface and of no interactions between the object of interest and the distribution of unwanted objects. These assumptions allow, as illustrated in this letter, the solution to the buried object detection problem within the framework of exact electromagnetic field scattering theory and the derivation of a computationally efficient optimum detection algorithm. More specifically, the letter is organized as follows. Section II is devoted to statement of the basic field equations governing the interaction of probing fields with the air/soil/object environment and derivation of a field translation property. Section III addresses the buried object detection problem and presents the maximum likelihood algorithm for the solution to it. Finally, Section IV is a discussion of the results of the letter with suggestions for possible future research avenues.

\section{CONFIGURATION AND SCATTERING EQUATIONS}

Consider the configuration in Fig. 1 in which a planar interface separates air (medium 1) from soil (medium 2). The orthogonal coordinate system $\mathbf{r}=\mathbf{x}+\hat{z} z$ is defined, with $\mathbf{x}$ indicating a two-dimensional (2-D) coordinate on the interface and the $z$ axis directed so that $z>0$ is air and $z<0$ is soil. Buried in the soil is a known target object, but its coordinates $\mathbf{r}_{c}=\mathbf{x}_{c}+\hat{z} z_{c}$ with $z_{c} \leq 0$ are unknown. A monochromatic plane wave with electric field $\mathbf{E}^{i}(\mathbf{r})=\mathbf{E}_{0} e^{i \mathbf{k}_{1} \cdot \mathbf{r}}$ and wavevector $\mathbf{k}_{1}$ is incident upon the planar interface. As the incident field reaches the interface, it partially reflects back into air and partially refracts into the soil where it interacts with the buried object. The interaction produces a scattered field, part of which refracts back into air where it is measured.

The total field $\mathbf{E}\left(\mathbf{r} ; \mathbf{r}_{c}\right)$ at position $\mathbf{r}$ is given by

$$
\mathbf{E}\left(\mathbf{r} ; \mathbf{r}_{c}\right)=\mathbf{E}^{b}(\mathbf{r})+\mathbf{E}^{s}\left(\mathbf{r} ; \mathbf{r}_{c}\right)
$$

where $\mathbf{E}^{b}(\mathbf{r})$ is the electric field in the absence of the buried object (background field) consisting of the incident and the reflected field in air and the refracted field in soil and $\mathbf{E}^{s}\left(\mathbf{r} ; \mathbf{r}_{c}\right)$ is the scattered field due to the presence of the buried object at the unknown location $\mathbf{r}_{c}$

$$
\begin{aligned}
& \mathbf{E}^{s}\left(\mathbf{r} ; \mathbf{r}_{c}\right) \\
& =\left\{\begin{array}{cl}
\mathbf{E}_{2}^{s}\left(\mathbf{r} ; \mathbf{r}_{c}\right) \equiv \int_{z^{\prime}<0} \overline{\mathbf{G}}_{22}\left(\mathbf{r}, \mathbf{r}^{\prime}\right) \cdot\left[\mathbf{E}^{b}\left(\mathbf{r}^{\prime}\right)\right. & \\
\left.+\mathbf{E}_{2}^{s}\left(\mathbf{r}^{\prime} ; \mathbf{r}_{c}\right)\right] O\left(\mathbf{r}^{\prime} ; \mathbf{r}_{c}\right) d^{3} r^{\prime}, & z<0 \text { (soil) } \\
\mathbf{E}_{1}^{s}\left(\mathbf{r} ; \mathbf{r}_{c}\right) \equiv \int_{z^{\prime}<0} \overline{\mathbf{G}}_{12}\left(\mathbf{r}, \mathbf{r}^{\prime}\right) \cdot\left[\mathbf{E}^{b}\left(\mathbf{r}^{\prime}\right)\right. & \\
\left.+\mathbf{E}_{2}^{s}\left(\mathbf{r}^{\prime} ; \mathbf{r}_{c}\right)\right] O\left(\mathbf{r}^{\prime} ; \mathbf{r}_{c}\right) d^{3} r^{\prime}, & z>0 \text { (air). }
\end{array}\right.
\end{aligned}
$$

In (2), the object function $O\left(\mathbf{r} ; \mathbf{r}_{c}\right)$ is defined as

$$
O\left(\mathbf{r} ; \mathbf{r}_{c}\right)=k^{2}\left(\mathbf{r} ; \mathbf{r}_{c}\right)-k_{2}^{2}
$$

where $k\left(\mathbf{r} ; \mathbf{r}_{c}\right)$ is the complex wavenumber at point $\mathbf{r}$ and $k_{2}$ is the complex wavenumber of the soil. Equation (2) is the Lippmann-Schwinger equation for the scattering problem and maps the object function to the corresponding scattered field nonlinearly.

The dyadic Green functions $\overline{\mathbf{G}}_{12}$ and $\overline{\mathbf{G}}_{22}$ are defined for the source point in soil and the observation point in air and soil, respectively. Explicit expressions for them can be found in [2]; however, the critical information for this context is that both Green 